\title{
50 nm THICK ALUMINUM NITRIDE PIEZOELECTRIC NANO-PLATE RESONANT THERMAL DETECTORS
}

\author{
Z. Qian, S. Kang, V. Rajaram and M. Rinaldi
}

Northeastern University, Boston, Massachusetts, USA

\begin{abstract}
This paper reports on the first demonstration of ultra-fast (thermal time constant, $\tau<10 \mu s$ ) and high resolution (noise equivalent power, $\mathrm{NEP} \sim 4 \mathrm{nW} / \mathrm{Hz}^{1 / 2}$ ) thermal detectors based on $50 \mathrm{~nm}$ thick Aluminum Nitride (AlN) piezoelectric resonant nanoplates. Efficient piezoelectric transduction of a high frequency $(\sim 1 \mathrm{GHz})$ lateral-extensional mode of vibration in a $50 \mathrm{~nm}$ thick AlN nano-plate is demonstrated for the first time and exploited for the implementation of the smallest volume $\left(\sim 87 \mu \mathrm{m}^{3}\right)$ laterally vibrating piezoelectric NEMS resonators reported to date. The effects of volume scaling on quality factor, $Q$, temperature coefficient of frequency, $T C F$, and associated detector $N E P$ and $\tau$ are experimentally investigated.
\end{abstract}

\section{INTRODUCTION}

One of the most important requirements for the implementation of a high resolution and simultaneously ultra-fast MEMS/NEMS resonant infrared (IR) detector is the scaling of the resonator thickness [1]. In fact, the scaling of the device thickness, $t$, while maintaining the resonator static electrical capacitance, $C_{0}$, constant to a value larger than the substrate parasitic capacitance, inevitably yields an improved IR detector figure of merit: $F O M=1 /(N E P \cdot \tau) \propto(\eta \cdot T C F \cdot Q) /\left(C_{0} \cdot t^{2}\right)$, where $\eta$ is the IR absorption coefficient of the structure. AlN resonant thermal detectors as thin as $500 \mathrm{~nm}$ have been recently demonstrated showing excellent thermal detection capabilities (NEP $\sim 0.4$ $n W / H z^{1 / 2}, \tau \sim 20 m s, F o M \sim 125 n W^{1} s^{-3 / 2}$ ) [2]. Nevertheless, despite such a high performance (comparable to the one of other existing uncooled IR detector technologies) the size of the demonstrated MEMS resonant IR detectors is $\sim 1$ order of magnitude larger than the one of commercially available microbolometers. This relatively large detector size has fundamentally prevented this technology from addressing the growing needs of small pixel size IR cameras for both military and civilian applications. In this work, we show that, by scaling the resonator thickness to $50 \mathrm{~nm}$ it is possible to implement IR detectors with reduced lateral dimensions (down to $32 \times 28 \mathrm{\mu m}^{2}$ ), comparable to the ones of commercially available microbolometers, and characterized by a FoM 2 orders of magnitude higher than what previously achieved by MEMS/NEMS resonant thermal detector technologies.

\section{EXPERIMENTAL RESULTS}

In this work an ultra-thin $(50 \mathrm{~nm})$ and high quality $\left(F W H M<3^{\circ}\right)$ AlN piezoelectric layer is employed to form the resonant bodies of lateral-extensional mode resonators (Fig. 1) with 5 different lateral dimensions ranging from $60 \mu \mathrm{m}$ to $28 \mu \mathrm{m}$ (Fig. 2). Moreover, thicker devices $(200 \mathrm{~nm}$ and $500 \mathrm{~nm}$ ) for each size are built as references to prove the effectiveness of volume scaling in improving device performance. The electromechanical performance of both the $50 \mathrm{~nm}$ thick devices and reference devices were characterized (Fig. 3, 4), demonstrating that the scaling of the device thickness down to $50 \mathrm{~nm}$ allows to maintain sufficiently high values of device capacitance (low impedance) while simultaneously guaranteeing the achievement of high electromechanical performance $\left(f \cdot Q \sim 10^{12}\right.$ and $\left.k_{t}{ }^{2} \cdot Q \sim 10\right)$ comparable to that of $10 X$ thicker devices. Furthermore, the experiments demonstrate that the use of a $50 \mathrm{~nm}$ thick AlN layer yields higher $Q$ values when the lateral dimensions of the devices are reduced below $50 \mu \mathrm{m}$.

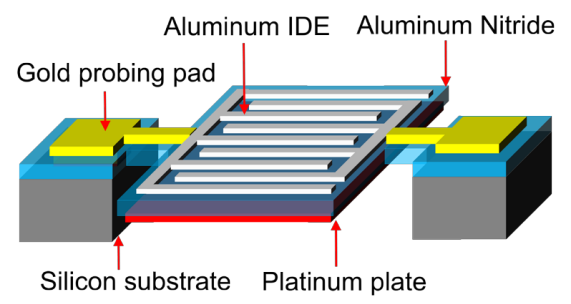

Figure 1: $3 D$ schematic representation of the AlN nano-plate resonant thermal detector.
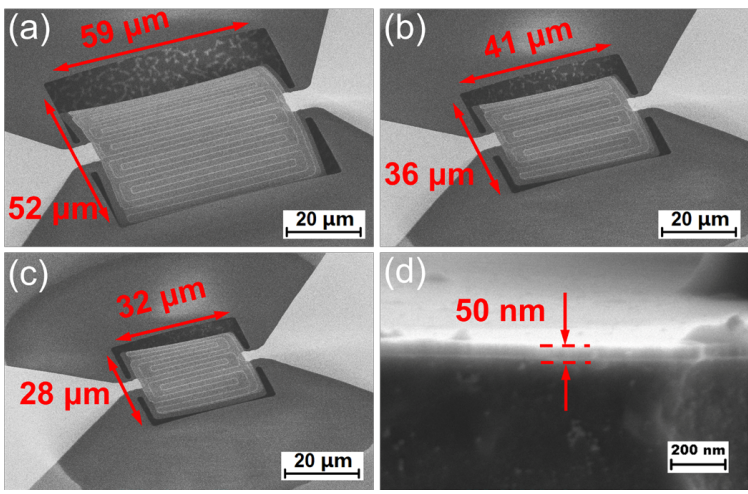

Figure 2: (a) to (c) SEM images of fabricated $50 \mathrm{~nm}$ AlN nano plate resonators with various lateral dimensions. Totally 5 different sizes were designed $\left(60 \times 68 \mu^{2}, 59 \times 52 \mu^{2}, 50 \times 44\right.$ $\mu \mathrm{m}^{2}, 41 \times 36 \mu \mathrm{m}^{2}$ and $\left.28 \times 32 \mu \mathrm{m}^{2}\right)$. The pitch, $W_{0}$, of the top IDE is fixed at $4 \mu \mathrm{m}$ for all devices. $t_{A l}=50 \mathrm{~nm}, t_{P t}=25 / 50 / 100 \mathrm{~nm}$ for $50 / 200 / 500 \mathrm{~nm}$ AlN and $t_{A u}=100 \mathrm{~nm}$. (d) A cross sectional view highlighting the $50 \mathrm{~nm}$ AlN thin film deposited on the Si substrate.

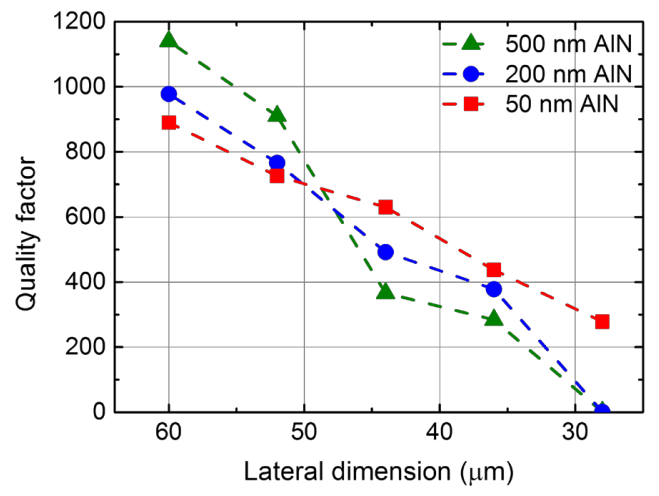

Figure 3: $Q$ factor values extracted from MBVD fitting of the fabricated scaled devices with various thicknesses of the AlN nano plate. For lateral dimensions below $50 \mu \mathrm{m}$, the $50 \mathrm{~nm}$ thick devices show higher $Q$ than the thicker ones. Low device capacitance and spurious modes prevent measurements of $Q$ values for the smallest $500 \mathrm{~nm}$ and $200 \mathrm{~nm}$ AlN devices. The extracted $k_{t}^{2}$ (without deembedding) of these devices varies between $1.1 \%$ and $0.7 \%$.
Solid-State Sensors, Actuators and Microsystems Workshop Hilton Head Island, South Carolina, June 5-9, 2016 
The thermal capacitances of the fabricated devices were estimated based on their geometries and material properties (Fig. 4) showing an ultra-low thermal time constant, $\tau \sim 2 \mu s$, for the smallest $50 \mathrm{~nm}$ thick device. The thermal resistances, $R_{t h}$ of the 50 $\mathrm{nm}, 200 \mathrm{~nm}$ and $500 \mathrm{~nm}$ thick devices were estimated based on their anchor geometries and material composition (not optimized in this work) and found to be $\sim 1 \times 10^{4} \mathrm{~K} / \mathrm{W}, 0.6 \times 10^{4} \mathrm{~K} / \mathrm{W}$ and $0.4 \times 10^{4}$ $K / W$, respectively. The $T C F$ of devices with three different thicknesses were characterized (Fig. 5), showing a high temperature sensitivity $\sim-52.5 \mathrm{ppm} / \mathrm{K}$ for the $50 \mathrm{~nm}$ devices (due to the more significant contribution of the metal electrodes characterized by a relatively large temperature coefficient of Young's modulus). These unique features were exploited for the first experimental demonstration of ultra-fast and high resolution AIN NEMS resonant IR detectors. The detectors were characterized using a Pendar Technologies $5 \mu \mathrm{m}$ quantum cascade laser and the experimental setup shown in Fig. 6. The frequency response to a modulated IR radiation of a $50 \mathrm{~nm}$ device was measured and compared to that of a $200 \mathrm{~nm}$ one (Fig. 7). The intrinsic absorption peaks of both the $50 \mathrm{~nm}$ and the $200 \mathrm{~nm}$ AlN devices are located at wavelengths much shorter than $5 \mu \mathrm{m}$ [3]. Therefore, they are both characterized by small and comparable values of IR absorptance, $\eta$, at $\sim 5 \mu \mathrm{m}$. The measurement results show a $2 X$ improvement in responsivity $\left(S \propto \eta \cdot R_{t h} \cdot T C F\right)$ for the 50 $\mathrm{nm}$ device in agreement with the analytical predictions. The frequency noise spectral densities, $f_{n}$, of the two devices were extracted from the measurements and the $N E P$ values were then calculated $\left(N E P=f_{n} / S\right.$, assuming $\left.\eta=1\right)$ to be $\sim 4 n W / H z^{1 / 2}$ for the $50 \mathrm{~nm}$ device and $\sim 6 \mathrm{nW} / \mathrm{Hz}^{1 / 2}$ for the $200 \mathrm{~nm}$ one. It is worth noting that, these values can be easily reduced to the $p W$ range by optimizing the anchor design [2]. The response times of the two devices were estimated $\left(\tau=C_{t h} \cdot R_{t h}\right)$ to be $\sim 10 \mu \mathrm{s}$ for the $50 \mathrm{~nm}$ device and $\sim 16 \mu \mathrm{s}$ for the $200 \mathrm{~nm}$ one. These experimental results demonstrate that the scaling of the resonator thickness enables the implementation of resonant IR detectors with largely improved detection capability $\left(\right.$ FoM $\left.\sim 2.5 \times 10^{4} n W^{1} s^{-3 / 2}\right)$, smaller form factor and reduced electrical impedance.

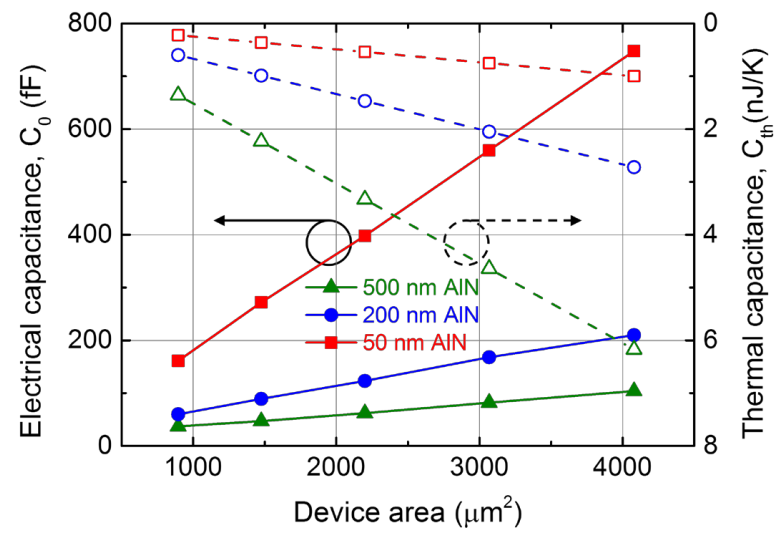

Figure 4: Measured device capacitances and estimated thermal capacitances as functions of the device area. When the device capacitance is smaller than $100 \mathrm{fF}$, it becomes comparable to the substrate parasitic capacitance deteriorating the effective electromechanical coupling of the resonant element (higher electrical impedance and insertion loss). Thermal capacitances are estimated based on the geometry and material properties of each device. $C_{\text {th }}$ as low as $0.2 \mathrm{~nJ} / \mathrm{K}$ is estimated for the smallest $50 \mathrm{~nm}$ device, rendering an ultra-low thermal time constant of $\sim 2 \mu$ s.

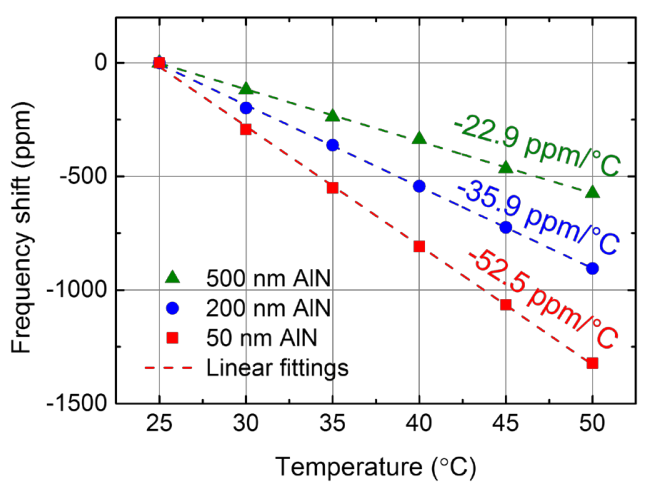

Figure 5: Measured TCF for devices with different thicknesses.

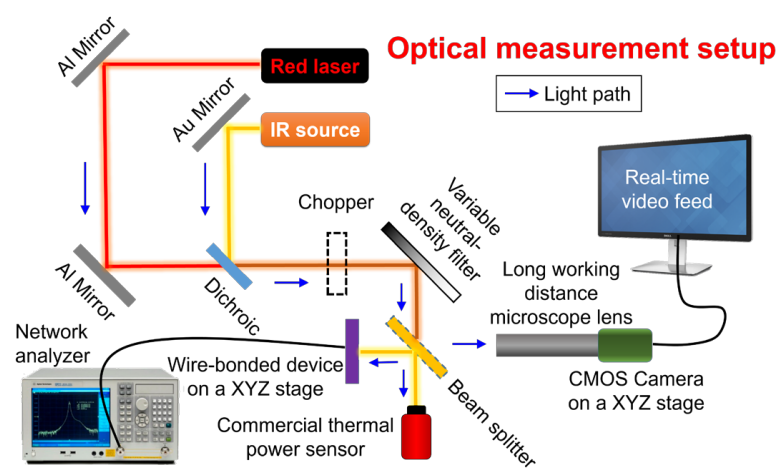

Figure 6: Experimental setup for infrared sensing measurements.

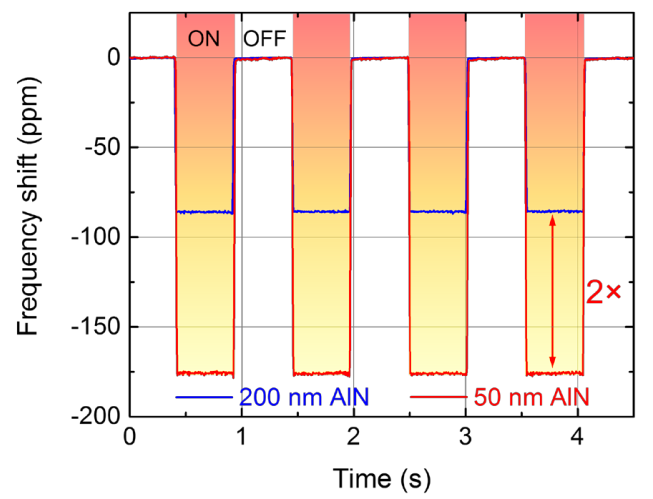

Figure 7: Measured frequency responses of a $50 \mathrm{~nm}$ device and a $200 \mathrm{~nm}$ reference with same lateral dimensions $\left(60 \times 68{\left.\mu \mathrm{m}^{2}\right)}^{2}\right.$ when exposed to a $5 \mu \mathrm{m}$ IR radiation modulated at $1 \mathrm{~Hz}$ by a chopper.

\section{REFERENCES}

[1] Y. Hui and M. Rinaldi, "Aluminum Nitride Nano-Plate Resonant Infrared Sensor with Self-Sustained CMOS Oscillator for Nano-Watts Range Power Detection”, Proc. IFCS 2013, pp. 62-65.

[2] Y. Hui, Z. Qian, G. Hummel and M. Rinaldi, "Pico-Watts Range Uncooled Infrared Detector Based on a Freestanding Piezoelectric Resonant Microplate with Nanoscale Metal Anchors", Proc. Hilton Head 2014, pp. 387-390.

[3] Z. Qian et al., "Graphene Aluminum Nitride NEMS Resonant Infrared Detector", under review.

\section{CONTACT}

Z. Qian, qian@ece.neu.edu; M. Rinaldi, rinaldi@ece.neu.edu 